\title{
Diagnosing Post Traumatic Stress Disorder: Using a Physiological Computing Method and Apparatus
}

\section{Michael Workman}

Chief Research Scientist and Director of Advanced R\&D, Security Policy Institute, Melbourne, Florida, USA

\begin{abstract}
Background: Post traumatic stress disorder (PTSD) is typically diagnosed using self-reports and physician interviews and observations. For example, the US military has used the Warrior Administered Retrospective Causality Assessment Tool (WARCAT), which is a self-report method for diagnostics. Methods: To help correct for associated deficiencies in self-reports, we utilized a computerized dual-task method and central nervous system (CNS) monitoring of reactions by participants who viewed troubling scenes.

Results: We found evidence to support unreported PTSD reactions to these incidents. Furthermore, we used a computerized technique to induce cognitive load to distract participants from the purpose of the assessment to gain more accurate assessments.

Conclusion: We conclude that using a computer apparatus is more effective in diagnosing PTSD than psychological instrumentation.
\end{abstract}

Publication History:

\section{Introduction}

At first glance, a study of post traumatic stress disorder may appear orthogonal to the topic of computer engineering; however, the subject provides an opportunity to demonstrate how computer technology may serve in addressing important social and psychological problems. In this study, we illustrate how computer technology may improve upon human processes and performance in both diagnostics and treatment.

Post traumatic stress disorder (PTSD) is classified in the Diagnostic and Statistical Manual of Mental Disorders, fourth edition, (DSMIV, American Psychiatric Association) as a nervous disorder, and it has become a widely recognized personal and social debilitation. The consequences of this disorder range from extreme detachment to extreme rage that can result in violent behaviors including suicide and murder. More commonly however, PTSD sufferers show severe social-dysfunction, including problems related to self-medication, difficulties holding down jobs, and extreme anxiety and depression [1].

It is important to note that not everyone who has experienced trauma or endures systematic abuse will develop PTSD [2]. The majority of the population eventually rebounds after tragic life events. However, for those people who lack this resilience, early diagnosis and intervention is critical to their long-term prognosis. Likewise, people have limits in their abilities to cope with systematic abuse, and consequently, even resilient persons may develop PTSD given the situation or situations. More accurate assessment will help lead to earlier interventions and better treatments for PTSD sufferers $[3,4]$.

\section{Background}

Since the 1990s, researchers c.f. [5] have been calling for better ways to assess PTSD rather than self-report, such as that captured in the military with the Warrior Administered Retrospective Causality Assessment Tool, or WARCAT [6]. A review of the PTSD literature by [7] noted several gaps, including: (1) differences in diagnoses among self-report instruments, (2) silos between military and civilian aspects of PTSD, (3) silos between government agencies, and (4) differences in manifestations of PTSD connected with head trauma (e.g. IED blast incidents) versus psychic trauma (e.g. episodic, such as rape or witnessing a savage brutality) versus systematic abuse (such as protracted and enduring psychological torture or psychologically abusive behaviors aimed at a victim).

A website administered by the US Veterans Administration in 2014 confirmed that these gaps still exist (http://www.ptsd.va.gov). Moreover, most of the solutions suggested by the military have relied on the tie between traumatic brain injuries (TBI) with PTSD assessments. Yet, studies (such as by Lazarus and others, c.f. [8]) have shown that in the general population, psychic trauma including plural surgical episodes aimed at intervening in life-threatening illnesses, automobile accidents, rapes, assaults, and systematic physical and/ or psychological abuses, are more likely to lead to PTSD than is brain injury (for an informative summary, see [9]). Also, a study presented by [10] argued that each of these issues still exist, and calls for more research. We proposed to help fill the noted gaps by testing a new computerized method for PTSD diagnosis. With a new diagnostic technique, we targeted the under-reporting problem and problems related to self-report methods in both military and civilian populations. We also aimed at transcending PTSD situations and knowledge silos with ontology (knowledgebase) to aid in timely, more cost effective, and holistic diagnoses and treatments.

Making accurate predictions about these life events is difficult from self-report data because of the social undesirability effects, and inability of the impaired to accurately report their conditions (indeed, many people are able to mask them). We determined that a physiological assessment method is needed for better assessments. Drawing from methods used by Lazarus, and studies by $[8,11]$ and [12] for assessing stress responses to disturbing stimuli, we present

"Corresponding Author: Dr. Michael Workman, Chief Research Scientist and Director of Advanced R\&D, Security Policy Institute, Melbourne, Florida, USA; E-mail: workmanfit@yahoo.com

Citation: Workman M (2017) Diagnosing Post Traumatic Stress Disorder: Using a Physiological Computing Method and Apparatus. Int J Comput Softw Eng 2: 118. doi: https://doi.org/10.15344/2456-4451/2017/118

Copyright: (@) 2017 Workman. This is an open-access article distributed under the terms of the Creative Commons Attribution License, which permits unrestricted use, distribution, and reproduction in any medium, provided the original author and source are credited. 
experimental evidence of accurate PTSD assessment, and make recommendations for a computerized physiological diagnostic apparatus.

\section{Post-Traumatic Stress Disorder (PTDS) and Physiological Computing}

Diagnostics for PTSD for the most part currently involve selfreporting and physician interviews and observations, as noted earlier. Based on these techniques, evaluators judge the inferred or observed propensities for deviant behaviors and criminality along ten incremental dimensions: (1) over-reactions to provocative stimuli; (2) regularly "acting out" in socially inappropriate ways; (3) excessive selfmedication for purposes of nullifying a precursor to negative thoughts (4) self-destructive tendencies; (5) extreme withdrawal behaviors; (6) co-morbidity with other pathologies such as with agoraphobia; (7) suicidal ruminations; (8) suicide attempts; (9) intentional violence, and (8) "black-out" violent actions including assaults and murders $[13,14,15]$. These conditions are important to incorporate into the assessments, as it may include a bimodal stimulus that trigger myriad of responses $[16,17]$.

\section{PTSD Assessment}

Arguably, traumatic brain injury (TBI) is a major source of PTSD in the military (c.f. [18]). This area is being actively researched [18], and many publications have addressed this problem (particularly in relation to concussions -including from non-combat situations such as sports - football and boxing). Also, genetic conditions including high function autism have been associated with PTSD-like symptoms [19-22]. We did not attempt to duplicate this work.

Although some anxiety-related behavior may be co-morbid with genetic conditions or as the result of TBI, importantly, PTSD has many sources that differentiate it from other nervous disorders. For instance, in cases where TBI is absent PTSD may result over a period of time from a systematically abusive or captive situation, or an "experienced" episode that rises to the level of a significantly emotional event $[14,23]$, which may or may not involve a traumatic brain injury. Or, PTSD may result from a combined series of different physical and/or emotional traumas (hybrid-trauma) sustained over a period of time [1]. Each of these conditions present challenges to extant PTSD assessments.

Non-brain-injury is a more common source of PTSD in the civilian population compared to war veterans, although it may also comprise significant membership within the military and intelligence communities as field actors witness or experience many assaults and cognitive insults [24]. It is important to note that people are not equally susceptible to PTSD. As [25] described about clinical depression, the majority of the population eventually rebound to homeostasis after a tragic life event. For those people who lack this resilience, early diagnosis and intervention is critical to their long-term prognosis.

Likewise, people have limits in their abilities to cope with systematic abuse $[9,24,26,27]$. Many of the solutions offered to address PTSD include exposure therapy vs. virtual reality simulations and fMRI (c.f. [28]). Nevertheless, these have been deemed by many practitioners as impractical and/or too expensive for most diagnostic situations, especially in the "field" [18]. We aimed at creating more accurate and more cost effective methods and applications for early intervention.

\section{PTSD and Physiology}

Behavioral descriptions of PTSD in the literature range from exhibitions of extreme indifference to extreme rage [26], but prior to these behavioral manifestations (which might be too late to prevent a tragedy), people manifest antecedent physiological changes such as pupil dilation, sweating, increased cognitive activity in the amygdale, prefrontal cortex, and in the limbic system, and changes in heart rate stemming from an episode that may trigger a behavioral manifestation $[8,25]$. Many of the solicitations for research have focused on applications that dedicate effort to the identification, collection, storage, and use of clinically relevant biomarkers to advance diagnosis, prognosis, and treatment of PTSD. Although we understand the interest in and the desire for biological markers of PTSD, we believe that a more feasible method is immediately available, while a longerterm solution may loom on the horizon.

Among the objectives in the literature are to significantly advance treatment strategies for PTSD including interventions for early, chronic, and latent onset cases. However, before a "leap" may be made into the completely biological realm, other methods of diagnosing PTSD may present a realistic and inexpensive interim procedure (For example, see [29] - which presents a behavioral analogue of a biologic conception in social contagions). At the least, at some point, biological indicators must be verified in material behaviors [30].

To elaborate on this concept, a central nervous system (CNS) monitor was used by [8] to detect "horror" affect levels at the sight of genital mutilations and wood shop accidents and other evocative incidents. From these, he and his colleagues were able to accurately predict normal and abnormal reactions when compared to a normal distribution. They were also able to explain the response according to cognitive schema for appropriate reactions versus disproportionate reactions using CNS output over a normal distribution.

We chose to build upon this technique by utilizing a dual-task cognitive test and monitoring with electroencephalography (EEG) and heart rate activity using electrocardiogram (ECG/EKG). Moreover, using the dual-task deception method for measurement, the social desirability of self-report would in theory be eroded.

\section{Method}

Extending from a grant-funded study (authors) we implemented a computerized dual-task test to induce cognitive load, along with measures that included (1) participant perceptions, (2) rater observations, and (3) physiological measurement (EEG/EKG). A dual-task test is one in which a stimulus is delivered while a participant performs a task. A common dual-task test is to have a participant receive an auditory stimulus (e.g. listening to a story narrative with headphones) while performing a writing task, which is measured such as by time and/or errors (called secondary and primary tasks, respectively). A common finding is that the participant will interrupt his/her writing task when his/her name is spoken in the story narrative.

Since cognitive functions are performed in various parts of the brain, the dual-task test can be used to determine (dissociate) the cognitive effort applied to tasks such as auditory tasks versus visual tasks. Alternatively, if the dual-task test exercises the same cognitive structures (e.g. a writing task and deciphering visual images) then the task serves to "load" or "overload" those cognitive structures. This 
Citation: Workman M (2017) Diagnosing Post Traumatic Stress Disorder: Using a Physiological Computing Method and Apparatus. Int J Comput Softw Eng 2: 118. doi: https://doi.org/10.15344/2456-4451/2017/118

Page 3 of 6

technique is useful when focusing on a result in which one does not want the participant to contemplate, but rather to react to the stimuli.

Next, exposure therapy is the most common protocol for treatment of PTSD. This therapy presents disturbing stimuli to PTSD sufferers until they become immune from the effects. Thus (with IRB approval, patient consent, and protections of HIPAA) we combined a dual-task test with exposure in the assessment activity.

For our exploratory study, we included four participants having been diagnosed with PTSD, and one control participant assessed as not suffering from PTSD. Given the assertions in the literature, we were interested in assessing a lack of physiological response as well as exaggerated response to the presentation of traumatic episodes. A secondary task served to induce cognitive load and act as a diversion (and a control variable) to elicit reaction rather than cogitation about the experiment. The experiment was designed to assess primary outcome measures: under and over-reaction to the presentation of violent visual and audio stimuli relative to a normal distribution, in particular, relative to a population diagnosed with PTSD and those without PTSD. Physiological measures were to be triangulated with physician observer measures.

As mentioned earlier, it is important to note that [31] used dual-task tests for cognitive process dissociation. Process dissociation is used to dissect which (and how much) cognitive resources are allocated to various stimuli and cognitive processing. However, to induce cognitive load, we set out to overload the same cognitive structures following the advice of [32] and [33] for what is known as a nonstandard paring (e.g. a working memory secondary task combined with a primary visual task, which according to [34], both use visualspatial cognitive structures). The secondary task used a computerized presentation of the stimulus dot pattern to induce cognitive load (as opposed to process dissociation, hence the non-standard pairing). The computer rendered stimulus was presented for $900 \mathrm{~ms}$ [35] -this is long enough to visually encode but not long enough to store in long-term memory [36]. Participants were told to keep this pattern in (working) memory for later recall.

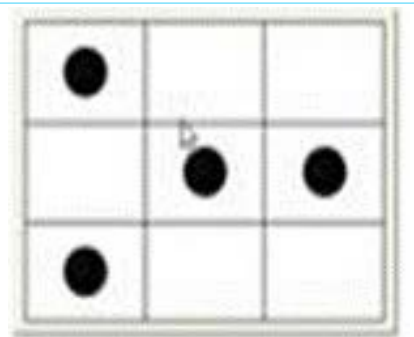

Figure 1: Example of Dot Pattern.

The primary task (which follows the secondary task stimulus) presented narrated video clips of accidents building on those done by [8] of wood shop accidents, automobile accidents, and genital mutilations. Unlike the [8] studies in which both clinical and "traumatized" narrations were used to gauge stress, we used only traumatized narrations to try to evoke as much of a response as possible.

Participants were then asked for a self-report reaction about the anxiety they experienced after each rendering (on a scale of 10 , low to high) while they were simultaneously monitored by an attending physician (EKG, EEG, blood pressure, and observations of blushing, sweating, etc.).

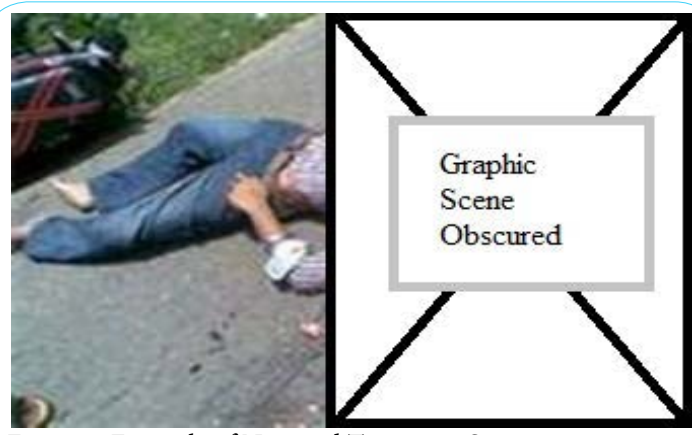

Figure 2: Example of Narrated Traumatic Scene.

From this scale (Figure 3 and Figure 4) we were interested in determining how participant's perceived their stress and anxiety levels compared to what was detected by clinicians from the CNS indicators in terms of stress and anxiety. Participants were then presented with a group of patterns to match (in each iteration), as seen in Figure 5. This secondary task is used as both a deception in the study purpose and to control for cogitation and memorization errors.

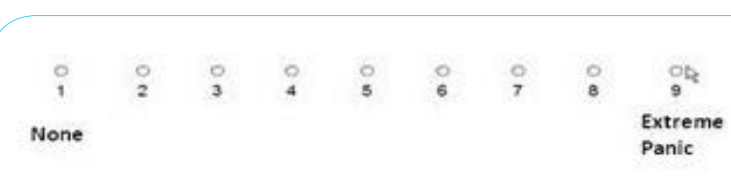

Figure 3: Anxiety Rating Scale.

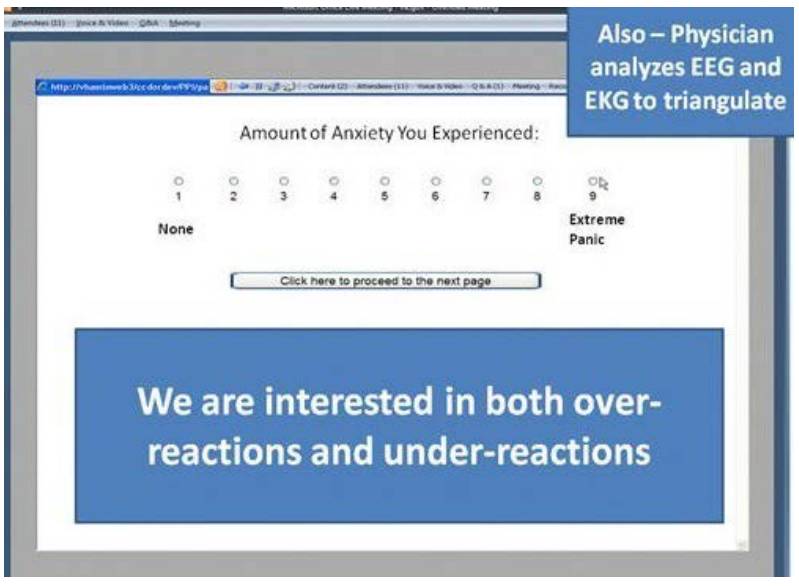

Figure 4: Anxiety Rating Scale Explained.
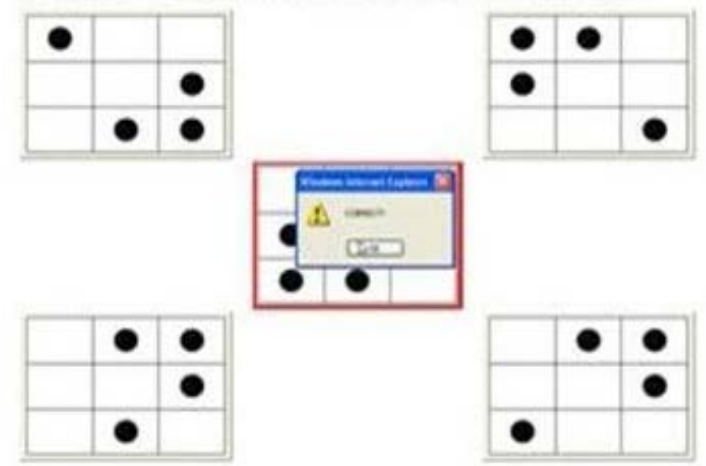

Figure 5: Example of Matching Task. 


\begin{abstract}
Results
We will now describe our results and explain a physiological computing method and apparatus for use in further PTSD study, taking into account the exploratory and opportunistic nature of the assessment we have presented. Our main purpose here is to indicate some possible outcomes using the method as explained, and to highlight some next steps for subsequent research, along with our recommendations for "field" applications. There were four participants having been diagnosed with PTSD using self-report and observations, and one participant diagnosed as without having PSTD symptoms using the same methods. A series of pretests (including resting heart rate and blood pressure) were done prior to the study assessments. These tests established CNS baselines (levels of voltage fluctuations resulting from ionic current flows in the brain, and in terms of heart rate and rhythm, including QRS complex).
\end{abstract}

\section{Non-PTSD Comparative Control Condition}

Study 1 was conducted with a male participant, age 31 , who was assessed as not suffering from PTSD symptoms or PTSD-related thoughts or ruminations by a board certified psychiatrist who had extensive experience in assessing PTSD and related disorders. A test with 35 iterations of the dual task test with task-narrated scene-task was given using the method previously described, and was monitored by a board certified cardiologist. The participant showed some elevated CNS levels from the baseline, but his heart rate was within normal limits. The pretest (from the baseline) pulse rate was $=68.00, \mathrm{sd}=4.04$, and the narrated scenes pulse rate was $\mathrm{m}=86.66, \mathrm{sd}=5.88(\mathrm{t}=-9.23$, $\mathrm{p}<.001$ ), which was significantly elevated. Moreover, the participant self-reported a moderately high feeling of anxiety produced by the narrated scenes $(\mathrm{m}=6.00, \mathrm{sd}=0.20)$. We drew the inference from this case that persons without PTSD should both experience and perceive some moderate levels of distress from the narrated scenes.

\section{PTSD Diagnosed Condition}

Study 2 was conducted with a female participant, age 44, who had been diagnosed with PTSD by a board certified psychiatrist who had extensive experience in assessing PTSD and related disorders. The assessment involved 35 computerized iterations of task-narrated scene-task, using the method previously described, and monitored by a board certified cardiologist.

The participant's CNS baseline was compared to the study results, which were elevated in the test. The pretest pulse rate was within normal limits: $\mathrm{m}=73.01$, $\mathrm{sd}=4.14$, but rose above normal limits when narrated scenes were presented. Narrated scene pulse rate was $\mathrm{m}=111.66$, with $\mathrm{sd}=10.71(\mathrm{t}=-14.24, \mathrm{p}<.0001)$. The participant reported strong feelings of anxiety produced by the narrated scenes $(\mathrm{m}=8.0$, sd $=0.56)$. This case was concordant with the previous diagnosis of PTSD.

Study 3 was conducted with a male participant age 29 previously diagnosed with PTSD by the same board certified psychiatrist, again with 35 computerized iterations and monitored by the same board certified cardiologist. Again the participant showed elevated CNS levels from the baseline. The pretest pulse rate was within normal limits: $\mathrm{m}=71.01$, $\mathrm{sd}=3.77$, but rose above normal limits when scenes were presented, pulse rate $\mathrm{m}=118.48, \mathrm{sd}=9.11(\mathrm{t}=-13.16, \mathrm{p}<$ $.0001)$. The participant reported relatively strong feelings of anxiety produced by the narrated scenes $(\mathrm{m}=7.5, \mathrm{sd}=0.43)$. This case was also concordant with the previous diagnosis of PTSD.
Study 4 was conducted with a male participant age 48 . The same test with 35 iterations was given using the method previously described, and again monitored by a board certified cardiologist. The participant showed nominal CNS level differentials from the baseline, and heart rate was within normal limits. The pretest pulse rate $=84.05$, $\mathrm{sd}=$ 5.09 , narrated scenes pulse rate was $\mathrm{m}=86.01$, $\mathrm{sd}=3.12(\mathrm{t}=-1.10, \mathrm{p}$ $>.05)$. The participant self-reported feeling a moderate to high level of anxiety produced by the narrated scenes $(\mathrm{m}=5.75$, sd $=0.09$ ). This case was discordant from the previous PTSD diagnosis in that the CNS results indicated little or no reaction while the self-report indicated some significant distress.

Study 5 was conducted with a male participant age 35 . Again, using 35 computerized iterations of the test, the participant showed elevated CNS levels from the baseline. The pretest pulse rate was within normal limits: $\mathrm{m}=69.55$, $\mathrm{sd}=4.00$, but rose above normal limits when narrated scenes were presented, narrated scene pulse rate $\mathrm{m}=112.01$, $\mathrm{sd}=10.16(\mathrm{t}=-15.44, \mathrm{p}<.0001)$. However, the participant reported only a mild feeling of anxiety produced by the narrated scenes $(\mathrm{m}$ $=3.0, \mathrm{sd}=0.18$ ). This case was discordant from the previous PTSD diagnosis in that the CNS results indicated a significant difference in CNS responses but self-reported little distress.

The most important suggestion from our exploratory study was that PTSD may have two modes - (1) an under reaction (psychic distance) to traumatic episodes that may eventually lead to an explosive event owing to pent up rage that went undetected, versus (2) consistent over-reaction, which is most often assessed and observed in current evaluative methods. It may also suggest that there may be misdiagnoses owing to the problems of self-reported conditions with high socially undesirable consequences.

Among our limitations is we acknowledge that using only four PTSD cases and one control case in our study was inadequate to make assertions about the efficacy of the computerized method compared to self-report and observation. However, combined with the theoretical foundation we established, we believe that there is sufficient evidence to warrant further study using directional hypotheses. For example, using self-report methods, it has been estimated that between 10-18\% of the population of the United States suffers from PTSD [37, 38]. However, according to other sources (c.f. $[24,39])$ these estimates may be quite low due to under reporting and social undesirability effects of self-report methods [10]. Moreover, these percentages are estimated to be relatively small compared to many other countries such as in South East and East Asia, South America, Africa, Europe, and the Middle East where there are significant kinetic conflicts [3].

Using CNS monitoring combined with computerized dual-task test for inducing cognitive load presents a low-cost and effective means to observe the effects of states of mind, including precursors to maladaptive behaviors that result from PTSD. A CNS monitor and application for this purpose can be deployed on a typical laptop computer with a few adaptations to collect sensory input (as illustrated by our prototype). Based on our results from our initial effort, we believe that it is possible to create a more accurate, pragmatic, generalized, and cheaper diagnostic for PTSD than currently exists (e.g. using the WARCAT) or than those that have been proposed (e.g. using fMRI). Moreover, the collection from a body of test results may be used to create a knowledgebase (ontology) of vital signs (and signatures) and responses related to PTSD for use in the medical community, similar to those currently being used and developed for the medical community regarding physical symptoms and illnesses. 
Citation: Workman M (2017) Diagnosing Post Traumatic Stress Disorder: Using a Physiological Computing Method and Apparatus. Int J Comput Softw Eng 2: 118. doi: https://doi.org/10.15344/2456-4451/2017/118

Page 5 of 6

Beyond this, creating PTSD ontology has the added property of being relational in so far as current (and future) technologies are able to reason over the relationships to develop both deductive and inductive inferences (e.g. using Bayesian Belief Networks) about what should be done given certain circumstances. This would be particularly important given that the PTSD responses appear to be bimodel between those who under react until they reach a tipping point and explode, versus those who consistently overreact until they selfdestruct. Knowing these features could help prevent disasters from PTSD-related behaviors.

\section{Conclusions}

In sum, as noted by [40], in large part, important questions about psychic trauma and PTSD have not yet been addressed leaving a vacuum in clinical care. Self-report has been notoriously poor in rending accurate results about socially undesirable behaviors, and are also poor in self-diagnoses of strong negative affect or muddled cognition. We believe that a more effective technique with relatively inexpensive technology is available and could be used in military post-deployment assessments, by first responders, and by care givers more generally [41].

Next steps beyond this exploratory study should include the development of physiological "signatures" and triangulated with psychological assessments for persistence in a knowledgebase (ontology), using a vastly larger set of participants in a variety of conditions. The ontology may utilize the Resource Description Framework (RDF) so that the ontology could be easily imported into extant systems used by modern systems.

Extending beyond this knowledgebase, we would propose building a "field kit" diagnostic device with a "triage" application. The diagnostic would be composed of a portable CNS monitor and computer, along with an application that would utilize the ontology and an expert reasoning system to guide that triage and immediate treatment decisions. Finally, we would suggest a graphical user interface (GUI) on a "dashboard" to assist the diagnosticians to the most important priorities. These features should increase accuracy of PTSD assessment, and reduce costs of medical treatment.

\section{Competing Interests}

The authors declare that they have no competing interests.

\section{References}

1. Brewin CR, Andrews B, Valentine JD (2000) Meta-analysis of risk factors for posttraumatic stress disorder in trauma-exposed adults. J Consult Clin Psychol 6:8 748-766.

2. Aarts H, Gollwitzer PM, Hassin RR (2004) Goal contagion: Perceiving is for pursuing. J Pers Soc Psychol 87: 23-37.

3. Hoge CW, Terhakopian A, Castro CA, Messer SC, Engel CC (2007) Association of posttraumatic stress disorder with somatic symptoms, health care visits, and absenteeism among Iraq war veterans. J Pers Soc Psychol 164: 150-153.

4. Vasterling JJ, Proctor SP, Amoroso P, Kane R, Heeren T, et al. (2006) Neuropsychological outcomes of Army personnel following deployment to the Iraq War. JAMA 296: 519-529.

5. Foa EB, Riggs DS, Dancu CV, Rothbaum BO (1993) Reliability and validity of a brief instrument for assessing post-traumatic stress disorder. Journal of Traumatic Stress 6: 459-473.

6. Terrio HP, Nelson LA, Betthauser LM, Harwood JE, Brenner LA (2011) Post-deployment traumatic brain injury screening questions: Sensitivity, specificity, and predictive values in returning soldiers. Rehabil Psychol 56 26-31.

Int J Comput Softw Eng

ISSN: 2456-4451
7. Rose SC, Bisson J, Churchill R, Wessley S (2009) Psychological debriefing for preventing post traumatic stress disorder (PTSD), NY: John Wiley \& Sons.

8. Lazarus RS (1991) Progress on a cognitive-motivational-relational theory of emotion. Am Psychol 46: 819-834.

9. LeRoy S, Elixson EM, O’Brien P, Tong E, Turpin S, et al. (2011) Recommendations for preparing children and adolescents for invasive cardiac procedures: a statement from the American Heart Association Pediatric Nursing Subcommittee of the Council on Cardiovascular Nursing in collaboration with the Council on Cardiovascular Diseases of the Young. Circulation 111: 1568-1570.

10. Flouri E (2011) Post-traumatic stress disorder (PTSD): What we have learned and what we still have not found out. Journal of Interpersonal Violence 39: 1087-1127.

11. Lazarus RS, Alfert E (1964) Short-circuiting of threat by experimentally altering cognitive appraisal. J Abnorm Psychol 69: 195-205.

12. Tomaka J, Kibler J, Blascovich J, Ernst JM (1997) Cognitive and psychological antecedents of threat and challenge appraisal. J Pers Soc Psychol 73: 63-72.

13. Bridge JA, lyengar S, Salary CB, Barbe RP, Birmaher B, et al. (2007) Clinical response and risk for reported suicidal ideation and suicide attempts in pediatric antidepressant treatment, a meta-analysis of randomized controlled trials. JAMA 297: 1683-1696.

14. Charney DS (2004) Psychobiological mechanisms of resilience and vulnerability: Implications for successful adaptation to extreme stress. Am J Psychiatry 161: 195-216.

15. Philipps D (2010) Lethal warriors: Uncovering the tragic reality of PTSD. London: Palgrave.

16. Burgess DJ, Crowley-Matoka M, Phelan S, Workman M, Dovidio JF, et al. (2014) Physicians' decisions to prescribe opiods for chronic pain: Biases and treatment decisions. Pain Med 15: 965-974.

17. Starke K, Ludwig AC, Brand M (2012) Anticipatory stress interferes with utilitarian moral judgment. Journal of Judgement and Decision Making 17: 61-68.

18. Brenner L, Terrio A, Homaifar H, Gutierrez BY, Staves PM, et al. (2010) Neuropsychological test performance in soldiers with blast-related mild TBI. Neuropsychology 24:160-167.

19. Bauminger N, Shulman C, Agam, G (2003) Peer interac tion and loneliness in high functioning children with autism. J Autism Dev Disord 33: 489-507.

20. Muller J, Roberts JE (2005) Memory and attention in obsessive-compulsive disorder: A review. J Anxiety Disord 19:1-28.

21. Orsmond GI, Krauss MW, Seltzer MM (2004) Peer relationships and socia and recreational activities among adolescents and adults with autism. $J$ Autism Dev Disord 34: 245-256.

22. Watson PJ, Shalev AY (2005) Assessment and treatment of adult acute responses to traumatic stress following mass traumatic events. CNS Spectrum 10: 123-131.

23. Massey M (1987) A synopsis of what you are is what you were when: There are (basically) two ways to change. A white paper published by the University of Colorado (UCo Press), Boulder, $\mathrm{CO}$

24. Brewin C, Andrews B, Rose S (2003) Diagnostic Overlap between Acute Stress Disorder and PTSD in Victims of Violent Crime American. Am J Psychiatry 160: 783-785.

25. Joormann J, Siemer M (2011) Affective processing and emotion regulation in dysphoria and depression: Cognitive biases and deficits in cognitive control. Social and Personality Psychology 5: 13-28.

26. Kessler RC, Galea S, Gruber MJ, Sampson NA, Ursano RJ, Wessely S (2008) Trends in mental illness and suicidal tendencies after Hurricane Katrina. Mol Psychiatry 13: 374-384.

27. Pyszczynski T, Greenberg J, Solomon W (1997) Why do we need what we need? A terror management perspective on the roots of human social motivation. Psychological Inquiry 8: 1-20.

28. Friedlander L, Derocher M (2005) Neuroimaging studies of obsessivecompulsive disorder in adults and children. Clin Psychol Rev 26: 32-49. 
Citation: Workman M (2017) Diagnosing Post Traumatic Stress Disorder: Using a Physiological Computing Method and Apparatus. Int J Comput Softw Eng 2: 118. doi: https://doi.org/10.15344/2456-4451/2017/118

Page 6 of 6

29. Jones MB, Jones DR (1995) Preferred pathways of behavioural contagion. Clin Psychol Rev 29: 193-209.

30. Haldane JBS (1955) The cost of natural selection. J Genet 55: 511-524.

31. Jacoby LL (1991) A process dissociation framework: Separating automatic from intentional uses of memory. Journal of Memory and Language 30 : 513-541.

32. Brunken R, Steinbacher S, Plass JL, Leutner D (2002) Assessment of cognitive load in multimedia learning: Using dual-task methodology. Exp Psychol 49: 109-119.

33. Hazeltine E, Ruthruff E, Remingonton RW (2006) The role of input and output modality parings in dual-task performance: Evidence for contentdependent central interference. Cogn Psychol 52: 291-345.

34. Anderson JR, Ross BH (1980) Evidence against a semantic-episodic distinction. Journal of Experimental Psychology - Human Learning and Memory 6: 441-478.

35. Pollatesk A, Reichle ED, Rayner K (2006) Tests of the EZReader model: Exploring the interface between cognition and eye movement control. Cogn Psychol 52: 1-56.

36. Spielberger CD, Sarason IG, Strelau J, Brebner JMT (1991) Stress and Anxiety. NY: Hemisphere Press.

37. Adler LE, Ivins BJ, Helmick K, Warden D (2010) Neuropsychological test performance in soldiers with blast-related mild TBI. Neuropsychology 24 160-167.

38. Toth JP, Reingold EM, Jacoby LL (1994) Toward a redefinition of implicit memory: Process dissociations following elaborative processing and selfgeneration. J Exp Psychol Learn Mem Cogn 20: 290-303.

39. Steenkamp MM, Litz BT, Gray MJ, Lebowitz L, Nash W, et al. (2011) A brief exposure-based intervention for service members with PTSD. Cognitive and Behavioral Practice 18: 98-107.

40. Maguen S, Litz B (2012) Moral inquiry in vetrans of war. PTSD Researc Quarterly 23: 1050-1835.

41. Foa EB, Cahill SP, Boscarino JA, Hobfoll SE, Lahad M, et al. (2005) Social, psychological, and psychiatric interventions following terrorist attacks: recommendations for practice and research. Neuropsychopharmacology 30: 1806-1817. 